\title{
A Hybrid System with Petri Net and Fuzzy Theory
}

\author{
Rong-Hou Wu ${ }^{1}$ Yang-Han Lee ${ }^{2}$ Yih-Guang Jan $^{2}$ Hsien-Wei Tseng ${ }^{2}$ Ming-Hsueh Chuang ${ }^{2}$ \\ Jheng-Yao Lin ${ }^{2}$ Wei-Chen Lee ${ }^{2}$ His-Chun Tseng ${ }^{2}$ \\ ${ }^{1}$ Dept. of Computer \&Communication Engineering, St. John's University, rhwu@mail.sju.edu.tw \\ ${ }^{2}$ Department of Electrical Engineering, TamKang University, 692351157@s92.tku.edu.tw
}

\begin{abstract}
In this paper, we issue a hybrid system, which is integrated with the fuzzy system and Petri net models. We exploit the characteristics, imprecise or ambiguous information, of fuzzy theory to map the algorithm of crisp inputs and outputs, furthermore, model problems, product a nonlinear function, and final predict the next state transition of Petri net graphs. In order to quickly and precisely predict the result state, we use the Center of Area (COA) defuzzification method to infer the state transition for this hybrid system. Finally, we issue an example of washed-machine to illustrate this proposed hybrid system.
\end{abstract}

Keywords: Center of area (COA) defuzzification, state transition, hybrid system, fuzzy system, finite state machines (FSM), and Petri net graphs.

\section{Introduction}

Many new control theories and finite state machines (FSM) had been brought about and applied to control system, in the recently years. Among them, such as fuzzy theory was widely famous by means of the inference-engineer of logical mathematical model that was the one of theories to successfully predict the results of one system by uncertainty data, without previously to notify the clear conditions of system. On the other hand, Petri Nets (PNs) was used for system model analysis and development tool, and was combination of graphic and mathematic properties. Nowadays, They are applied in different kinds of model construction, analysis, and simulation.

Still now, lacking of the researches and papers integrates Petri net and fuzzy theory, and no common rules are defined of both. By aforementioned reasons, this paper will investigate how to exploit the fuzzy theory to infer the state transition in Petri net model, and the inference rules are embedded in the transition nodes of Petri net model to predict the transformation of transactions or tokens. Anywhere, we make the two different systems inference models as a new novelty graphic integrator in which the interpret inference rules of Fuzzy theory is build into the transition nodes of Petri net model to predict and analyze the moving tokens of process control systems.

In recent years, because of the change of sciences and technologies, make all kinds of systematic structures and functions complicating day by day. In the tradition, the analysis method of the complicated system is to find out the systematic structure prior to the systematic physical characteristic, and then utilize the physics formula to analyze the control-system with modeling. The control-system has already getting complicated and use the physics formula and systemmodel, which uses one layer analytic approaches of model (Model-Level analysis approaches) [2]-[4] and must input some materials to initial and get in the system, to describe [5][6].

For the output of result productions, which are the relation of analyzing, condition defining, output arrangement, structure systematically, and process reasonable. They are set up and performed by the algorithm, and modeled to control the systems with the proper mathematics, respectively. In addition, the behavior of the proposed discrete event system (DES) for modeling, predicting, and calculating is used the clear (crisp), precise, and confirmed (certain) characteristics of fuzzy theory. The meaning of clear (crisp) represents at binary logic, judge one of the binary logic that is distinguished only into 'denying' and 'really'. The meaning of accurate (precise) represents for the phenomenon and characteristics of a modeled system, which expresses with accurately parameters. The meaning of confirm that represents the structure and parameters of model via clear learning, no matter how the outputs do not have any queries. In the new era, this sciences and technologies are developed, the systems are becoming to complication and unable to use quantitative to analyze already, thus make the traditional system analytic approaches face the serious bottleneck.

The structure of this paper is organized as follows. In Section 2, we describe the characteristics of fuzzy logic and Petri net, individually. Section 3 illustrates the inference methods of place and transaction of the proposed system, in which combines to the fuzzy system and Petri net graphs. Finally, remark the conclusion in Section 4, in which summarizes the research results, and put forward the suggestion to the research direction in the future.

\section{Embedding Fuzzy Inference Engineer into Petri Nets}

The process of defining the Petri net graph involves two steps. First, we define the Petri net structure, which is analogous to the state transition diagram of 
automaton. Then, we adjoin to this graph an initial state, a set of marked states, and a transition labeling function [1]. Petri net structure is a weighted bipartite graph: $(P, T, F, w)$, where, $P=\left\{\mathrm{P}_{1}, \mathrm{P}_{2}, \ldots, \mathrm{P}_{\mathrm{n}}\right\}$ is the finite set of places, $T=\left\{\mathrm{T}_{1}, \mathrm{~T}_{2}, \ldots, \mathrm{T}_{\mathrm{m}}\right\}$ is the finite set of transitions, $F=(\mathrm{P} \times \mathrm{T}) \cup(\mathrm{T} \times \mathrm{P})$ is the set of arcs from places to transactions and from transactions to places, $\mathrm{w}: \mathrm{F} \rightarrow\{1,2,3, \ldots\}$ is the weight function on the arcs. In automata systems, the state transition mechanism is directly connected to the arcs in the state transition diagram. For state transition mechanism, the Petri net is provided by moving tokens through the net and hence changing the state. The state of Petri net is changed while a transaction fires or occurs. Then one token is moved from each of the input places to the one of output places.

The fuzzy systems are structured numerical estimators. They start from highly formalized insights bout the structure of categories found in the real word and then articulate fuzzy IF-THEN rules as a kind of expect knowledge.

In light of their similarities, Petri net and fuzzy systems are suitable for solving many of the same problems, achieving some degree of machine intelligent, and graphing the properties of a matrix relation of systems. The same characteristics of both are vertex graph to illustrate the state transition, relation matrix to identify the relation of input and output state equation, and the tree path to describe the state model.

In this paper, we will embed the fuzzy logic into the transition nodes (abbreviated to T) of Petri net to infer the state transfer to implement a hybrid intelligent system, which has the following characteristics: 1) the fuzzy set is convex, 2) the fuzzy numbers are defined by mixed function, which is combination of the $S$ function and $\pi$ function, 3) the fuzzy set-relation equations focus on max-min composition, $\mu_{\mathrm{X}}(\mathrm{y})=\mu_{\mathrm{YoR}} \quad(\mathrm{y})=\max \min$ $\left.\left[\mu_{\mathrm{X}}(\mathrm{x}), \mu_{\mathrm{R}}(\mathrm{x}, \mathrm{y})\right], 4\right)$ use the method of Center of area (COA) to defuzzy.

When drawing Petri net graphs, we need to differentiate between the two types of nodes, places ( abbreviated to P) and transitions. Among places and transitions are connected by an arc, we allow multiple arcs to connect two nodes and assign a weight to each arc representing the number of arcs, for instance Figure 1. A token is to move among two nodes from place (or transition) to transition (or place) while transaction happens, the moving path of token is decided to the inference rules of transition.

Refer to Figure 1, we construct the coverability tree of the Petri net model and its input/output matrix, both are shown in Figure 2.

\section{The Fuzzy Inference Engineer in Petri Net Graph}

The inference engineer of fuzzy system is embedded in the transaction node of this proposed Petri net to predict where the targets, i.e. the next places, of a moving token. For the proposed Petri net, in which the transition node with the function of inference engineer is achieved by fuzzy logic. We had divided the proposed Petri net into three levels, there are initial level, hidden level, and terminal level.

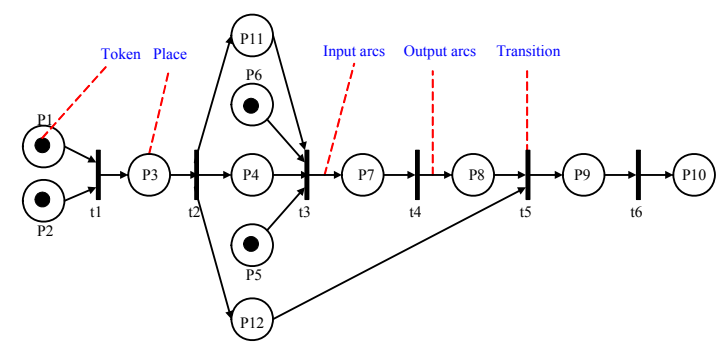

Fig. 1: The structure of Petri net.

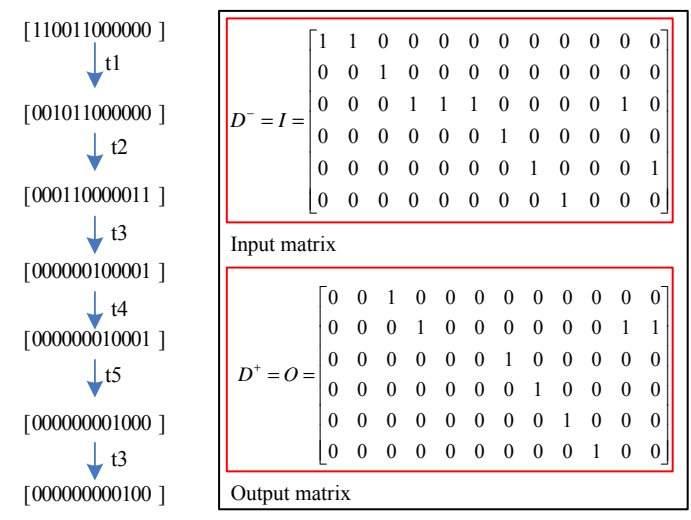

Fig. 2: Coverability tree and input/output matrix of the example of Fig1.

The initial level (also called the fuzzy level) is used to fuzzy the input variables, and combination of start node, which maybe place or transition. If the start node is place, which must be masked some tokens, then connected to transition node. If the start node is transition, which must be defined a fired condition by fuzzy logic, then connected to place node. The relation of place and transition of this Petri net mixed fuzzy theory in initial level is illustrated in Figure 3.

Second-level is hidden level (also called the inference level), which is combination of a serial of transition nodes and places nodes. The transitions is used to infer the next place (state) of a moving token, the inference rule is decided to the fuzzy logic of the system. The relation of place and transition of hidden level is illustrated in Figure 4. The set of input and output places from transition $\mathrm{T}_{\mathrm{i}}$ is represented to I (Ti) $=\{\mathrm{P} 1, \mathrm{P} 2, \ldots, \mathrm{Pn}\}$ and $\mathrm{O}\left(\mathrm{T}_{\mathrm{i}}\right)=\{\mathrm{P} 3, \mathrm{P} 4, \ldots\}$, where $\mathrm{i}$ $=1,2, \ldots, \mathrm{n}$, individually.

The inference process is progressed by transition Ti according to the fuzzy rules with the aND Boolean logic operation. The Producing output of each transition has different behalf of system parameter and 
attitude, which are turned into token that is the incident will gather and change each setting value and comparing in the attitude to excite. The token of Petri net represents the systematic signal parameter in each fuzzy value while assembled.

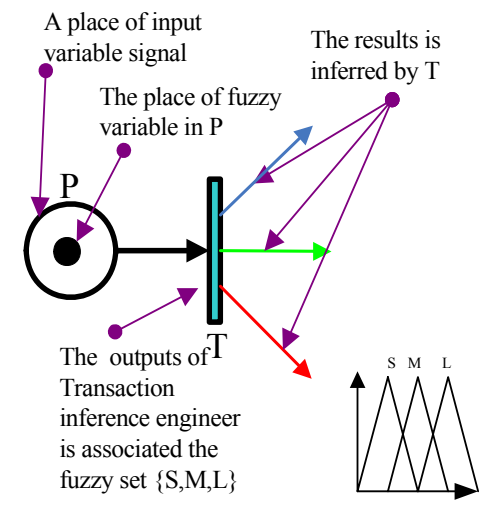

Fig. 3: The initial level of Petri net with fuzzy logic.

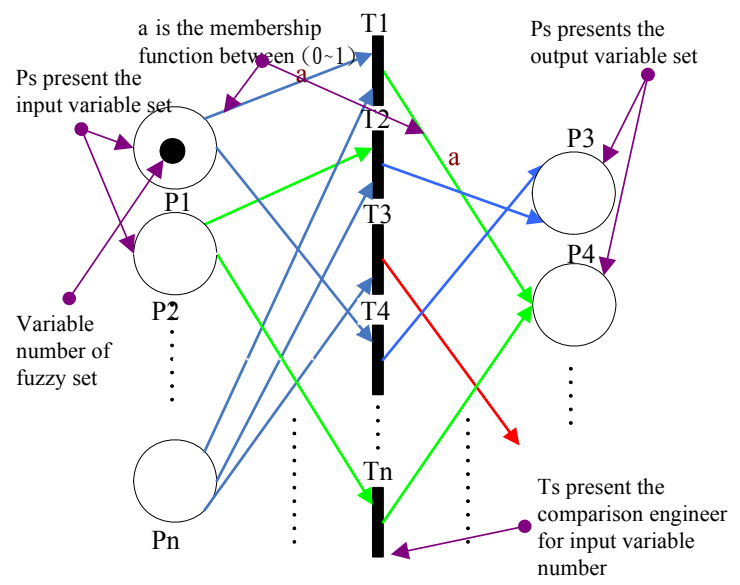

Fig. 4: The hidden level of proposed Petri net.

The third-level is the terminal level, which is used to store the results by defuzzing (shown in Figure 5). The set of output places from transition node $(\mathrm{T})$ is represented to $\mathrm{O}\left(\mathrm{T}_{\mathrm{t}}\right)=\{\mathrm{Pt}\}$.

In a word, the place is used to narrated state, transition is used to change the attitude becoming, and the token that can indicate every place after transiting of different levels in the above description.

Example: for a washing machine, the output variable of washing time (T) is depended upon two input variables, the weight of clothes $(\mathrm{X})$ and stream of water (Y). Those variables are departed three degrees, which are small (S), middle (M), and large (L) defined as follows:

- The weight of the clothes is $\mathrm{X}$, which range is from 0 to 10 (shown in Figure 6) and the unit is kilogram $(\mathrm{Kg})$.

- The stream of water is $\mathrm{Y}$, which range is from 0 to 80 (shown in Figure 6) the unit is liter per minute (1/min.).
- The washing time is $\mathrm{T}$, which range is from 0 to 100 (shown in Figure 6) and the unit is kilogram (min.).

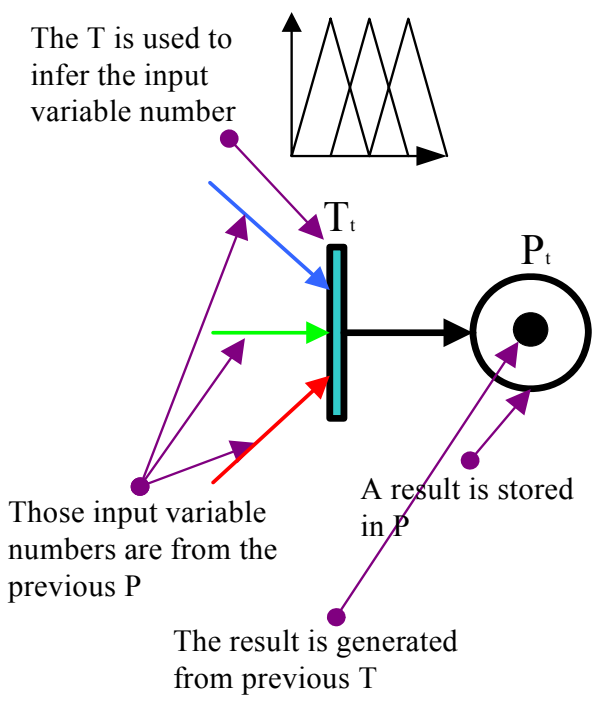

Fig. 5: Terminal level of proposal Petri net.
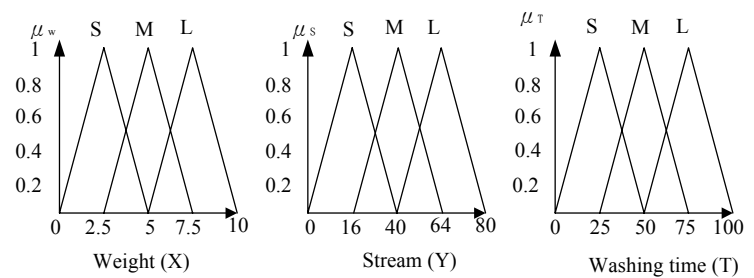

Fig. 6: Membership functions of W, Y and T.

The inference engineer is constructed of " If $<$ input variable $>$ and $<$ input variable $>$ THEN $<$ output variable $>$ " fuzzy rule. According to the able fuzzy rule, the relation of $\mathrm{X}, \mathrm{Y}$, and $\mathrm{T}$ variables are listed in Table 1.

Table 1: Fuzzy relation table of Example

\begin{tabular}{|c|c|c|c|c|}
\hline \multicolumn{2}{|c|}{ Washing time (T) } & \multicolumn{3}{c|}{ Weight (X) } \\
\cline { 3 - 5 } & S & M & L \\
\hline \multirow{3}{*}{ Stream (Y) } & S & M & L & L \\
\cline { 2 - 5 } & M & S & M & L \\
\cline { 2 - 5 } & M & S & S & L \\
\hline
\end{tabular}

If we want to wash the clothes, while turn on the power, the washing machine automatic detects the weight of clothes (X) is $3.2 \mathrm{Kgs}$, and adjusts the stream water $(\mathrm{Y})$ to $32 \mathrm{l} / \mathrm{min}$., individually. Refer to Figure 7, we get the input fuzzy set of $\mathrm{X}_{3.2 \mathrm{Kg}}$ and $\mathrm{Y}_{32}$ $1 /$ min. as follows:

The fuzzy set of $\mathrm{X}_{3.2 \mathrm{Kg}}=\{0.8 / \mathrm{S}, 0.2 / \mathrm{M}, 0 / \mathrm{L}\}$

The fuzzy set of $\mathrm{Y}_{32 \mathrm{l} / \mathrm{min}}=\{0.4 / \mathrm{S}, 0.8 / \mathrm{M}, 0 / \mathrm{L}\}$

For the above definition example, we assume and set the input value of $\mathrm{X}$ and $\mathrm{Y}$ to $3.2 \mathrm{Kg}$ and 32 liter, respectively. The initial nodes of the proposed Petri net graph are corresponding to the $\mathrm{P} 1$ and $\mathrm{P} 2$ place, 
individually. Furthermore, the $3.2 \mathrm{Kg}$ and 32 liter places fire the marks and been turned into the fuzzy value on state $\mathrm{P} 1$ (the weight of washed clothes, $\mathrm{X}$ ) and P2 (the water stream, Y) those are the first part of systems inputs at the beginning, respectively. Then the exciting attitudes have became more corresponding to the membership of the fuzzy function and correspond to next states of ownership by inferring the transaction nodes, T1 and T2. Sequentially, the fuzzy regular inference of the second part will turn into the exciting results, which are produced from the attitude of every second-part places of the first level. The tokens are delivered to the corresponding next place from the previous place via the connection line. For instance the changed $\mathrm{X}$, the product places of P3 (0.8/small), P4 (0.2/middle), and P5 (0/large) are derived from P1 place. In other words, the P6 (0.4/small), P7 (0.8/middle), and P8 (0/large) places are derived from $\mathrm{P} 1$ place of the changed $\mathrm{Y}$ variable parameter.

For the transaction nodes, $\mathrm{T}$, of the proposed Petri net graph are constructed with the capacity of inference. The embedded inference engineer is implemented with rule-based, which is defined as $I F<$ the crisp value of input $>$ THEN $<$ the corresponded memberships value of fuzzy set>. Then, a token is delivered to the corresponded place via the connection arc.

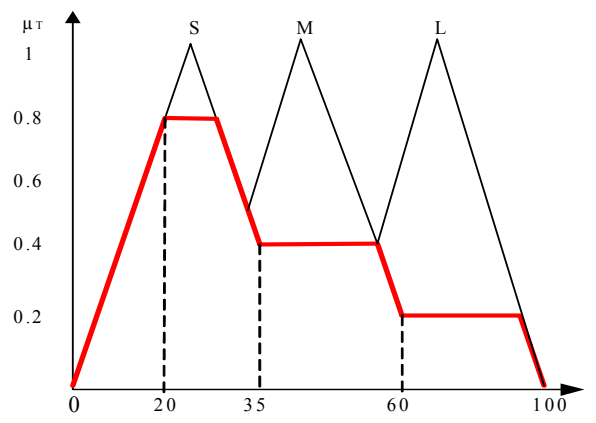

Fig. 7: The result of washing machine of example. $\mu$

The real washing time is defuzzied by the center of area (COa) defuzzification formula. The washing time is calculated as following steps, $\mathrm{z}_{\mathrm{COa}}=\Sigma \mu_{C}\left(\mathrm{z}_{\mathrm{j}}\right) \mathrm{z}_{\mathrm{j}}$ $/ \Sigma \mu_{C}\left(z_{j}\right)$, where $j=1, \ldots, n$, and $n$ is the number of quantization levels of the output, $\mathrm{z}_{\mathrm{j}}$ is the number of control output at the quantization level $j, \mu_{C}\left(z_{j}\right)$ represents its membership value in the output fuzzy set. Refer to Figure 7 and exploit the formula of COA, we get the fuzzy set of washing time as $w=\{0.8 / 20$, $0.4 / 35,0.2 / 60\}$, and calculate the washing time exploit COa formula, The washing time $(T)$ :

$T=\frac{T_{1}}{T_{2}}, T=41.025 \mathrm{~min}$

$T_{1}=5 \times .2+10 \times .4+15 \times .6+20 \times .8+25 \times .8+30 \times .8+35 \times .6+40 \times .4+45 \times .4+$ $50 \times .4+55 \times .4+60 \times .4+65 \times .4+70 \times .2+75 \times .2+80 \times .2+85 \times .2 \times 90 \times .2$ $+95 \times .2$

$T_{2}=.2+.4+.6+.8+.8+.8+.6+.4+.4+.4+.4+.4+.4+.2+.2+.2+.2+.2+.2$

\section{Conclusions}

In this paper, we attempt mixing two different characteristics theories, the fuzzy system and Peri net system, to create a novel Petri net graph, in which the transition nodes have the capability to infer and predict the state change. The aND logic operation is explicate to the fuzzy logic. To indicate that the proposal Petri net is usage, we also have created a Petri net system, which is a hybrid system for the Petri net and fuzzy system.

In this paper, we also have issued a completed novel Petri net, which implement the inference in transition with the fuzzy logic algorithm and have the Chinese graph user interface (GUI). In the future, we will attempt to embed the inference engineer into the place node of the Petri net system and exploit the Neuron network technology.

\section{Acknowledgements}

The authors would like to express their thanks to Ph.D. Yue-Ru Chuang for his helpful discussions. This work was supported by the National Science Council, Taipei, Taiwan, R.O.C. under Contract NSC 94 - 2213 - E 129 - 005, NSC 94 - 2213 - E - 032 - 005, NSC 94 2745 - E - 032 - 001 - URD, NSC 94 - 2745 - E - 032 004 - URD, and the funding from St. John's University and Tamkang Unversity for the UniversityDepartment joint research project.

\section{References}

[1] J. Bugarin, and S. Barro, "Fuzzy reasoning supported by Petri nets," IEEE Transactions on Fuzzy Systems, Vol.2, No.2, pp.135-150, 1994.

[2] S. M. Chen, J. M. Ke, and J. F. Chang, "Knowledge representation using fuzzy Petri nets," IEEE Transaction on Knowledge and Data Engineering, Vol. 2, No. 3, 1990, pp. 311319.

[3] G. Looney, "Fuzzy Petri nets for rule-based decision making," IEEE Transaction on Systems, Man, and Cybernetics, Vol. 18, No. 1, 1988, pp. 178-183.

[4] H. Scarpelli, F. Gomide, and R. Yager, "A reasoning algorithm for high level fuzzy Petri net," IEEE Transaction on Fuzzy Systems, Vol. 4, No. 3, 1996, pp. 282-294.

[5] Jih-Fu Tu, "Using Coloured Petri net for Modeling a High-Performance of Processor Communication," The Journal of St. John's university, September 2005, St. John's University, Taiwan, pp. 12-20.

[6] J. -F. Tu and Yin.-Tuei Hsu, "Exploiting Petri Net Graph to Model the FSM of Modulo-P Counter," 2004 IEEE Conference on CIS, Dec. 1-3, 2004, Singapore, pp. 1089-1093. 